\title{
Reaching the 'First 90': Proposal for Intervention Strategy in Order to Expanding Access to HIV Testing among Migrant and Vulnerable Populations
}

\author{
Ilaria Uccella ${ }^{1,2 *}$ \\ 1 INMP National Institute for Health, \\ Migration and Poverty, Rome, Italy \\ 2 Emergency Depertement-Ospedale \\ Santissima Trinità, Sora, Italy
}

\section{*Corresponding author: Ilaria Uccella \\ $\equiv$ ilaria.md@tiscali.it}

\section{Abstract}

Background: UNAIDS proposed a set of three targets that, if achieved, are predicted to end the AIDS epidemic by 2030 . The targets, known as 90-9090, call for $90 \%$ of people living with HIV (PLHIV) to know their status, $90 \%$ to receive antiretroviral therapy and $90 \%$ uptake of HIV tests. But insufficient data are reported on the estimated PLHIV prevalence on migrants and vulnerable populations across Europe. At the end to reduce this gap and to evaluate the behavioural characteristics of these populations, the HIV rapid test and counselling were offered inside a public health project.

Methods and findings: During the period December 2012-2013 all migrants and Italian people, aged between 16-70 years old, attending the infectious disease clinic of the National Institute for Health, Migration and Poverty (INMP) in Rome, Italy, were enrolled. HIV rapid testing was free and patients were asked to fill in a questionnaire evaluating knowledge about HIV/STIs, prevention methods, sexual behaviours and stigma. Patients with sexual risk behaviours or with a recent diagnosis of STIs were invited to come back after 3-6 months and a postcounselling questionnaire was offered. Out of the total sample, 99.2\% accepted the "rapid test"; 13 participants resulted positive $(1.45 \% ; 95 \% \mathrm{Cl}: 0.75-2.53)$ with 10 new diagnosis (1.22\%; $95 \% \mathrm{Cl} 0.58 \%-2.22 \%$ ). $46 \%$ answered the questionnaire and $18.6 \%$ completed the follow-up.

Conclusion: The approach providing HIV rapid test, associated to other instruments, such peer mediators inside the counselling setting, could be useful for the health care services and prevention programs to expanding access for vulnerable populations. But this approach reiterates the need of new policies toward marginalized populations at the aim to encouraging the access to health services and to refer to preventions programs.

Keywords: HIV; Rapid test; Counselling; Immigrants; Prevention; Questionnaire

Received: December 29, 2017; Accepted: January 22, 2018; Published: January 29, 2018

\section{Introduction}

A total of 4.7 million people immigrated to one of the EU-28 Member States during 2015, while at least 2.8 million migrants were reported to have left an EU Member State. In addition, 1.9 million people previously residing in one EU Member State migrated to another Member State [1].
Migrants are more likely to be exposed to social determinants of ill health as poverty, social exclusion, and unemployment, which can result in poor health-seeking behaviours [2-5].

Other structural factors, as legal and administrative status, health care and community-related barriers, can further prevent migrants from accessing health services, particularly HIV testing [6], access to treatment, and safer sex practices [7]. 
Previously it had been assumed that most HIV diagnosed among migrants in Europe was acquired in the country of origin, particularly those born in sub-Saharan Africa. Yet it is unclear whether infections are acquired pre- or post-migration [8].

In 2015, 29,727 cases of HIV were diagnosed and reported in the EU/EEA, resulting in a rate of 6.3 per 100,000 populations. The notification rate and the number of new HIV diagnoses reported have remained unchanged since 2011, with between 29,000 and 33,000 new cases reported annually (notification rates of between 6.3 and 6.5 per 100,000 populations). Despite evidence that HIV diagnosis is occurring earlier post-infection, the estimated number of people living with HIV (PLHIV) who were unaware of being infected in 2015 was 122,000 or $15 \%$ of all PLHIV ( $n=810,000)$ [9].

Among the 27 countries reporting on new HIV diagnoses and transmission consistently between 2005 and 2014, one third (37\%) of cases were foreign-born [10]. Furthermore some EU countries do not provide HIV care for undocumented people [11].

Although surveillance data show that people living outside their country of birth form a substantial proportion of the population affected by the HIV epidemic in Europe, limited data are available about migrant populations to inform policy and practice for these communities $[8,12]$.

Understanding barriers to accessing HIV care and post migration transmission several cross-sectional studies have sampled migrant populations, but these were often limited to migrants from one region (black Africans) [13] or Central and Eastern Europeans [14] or migrants in one country [15].

Within the European Network of HIV/AIDS Cohort Studies to Coordinate at European and International Level Clinical Research on HIV/AIDS (EuroCoord), the advancing Migrant Access to health Services in Europe (aMASE) study whose overall aim is to provide the evidence to prevent HIV infection and improve diagnosis and prognosis of migrant populations living with HIV in Europe in order to support policy development at the European level. The results of the study will improve the understanding of post migration transmission dynamics and the barriers to health care for migrants in Europe [16].

In Italy, in 2015 the proportion of new HIV cases among migrants is $28.8 \%$, increased from $15.4 \%$ in 2010 to $16.9 \%$ in 2014 in men, with an incidence of 18.9 compared to 4.3 per 100.000 in Italianborn people [17]. Further, among 3,059 late HIV presenters, $30.7 \%$ were immigrants [18]. No national data are reported regarding the number of immigrant tested, particularly undocumented, the HIV/STIs knowledge and prevention methods. On the bases of new scenario of migration flows in EU countries, that could influence the HIV/AIDS epidemiology in the coming years and to expand the access to HIV test and counselling to vulnerable populations, including migrants, we conducted a project that consisted of the HIV rapid test associated with multilingual written consensus and questionnaires and peer mediators trained inside the pre and post-counselling setting.

\section{Methods}

The National Institute for Health, Migration, and Poverty (INMP), Rome (Italy), is a national public institute providing free access to outpatient care, mainly to vulnerable populations including migrants (regularly staying and undocumented), homeless and Roma.

On February 2012, INMP started a project-funded by the Ministry of Health-aimed at promoting access to HIV testing and at disseminating knowledge about STIs.

This project was conducted in different steps: training of health care professionals, patients' enrolment, pre-test counselling, offer of HIV rapid test with informed consent and post-test counselling.

All subjects aged $16-70$ years old, attending the infectious disease clinic during the period December 2012-2013 were enrolled. Subjects who performed the test in the previous 6 months, without any risk referred or with previous HIV diagnosis, were excluded from the study.

HIV rapid test (Determine ${ }^{\mathrm{TM}} \mathrm{HIV}-1 / 2 \mathrm{Ag} / \mathrm{Ab}$ Combo-Alere) was offered for free [19]. After 20 min results were reported to the patient. If the test resulted reactive, a confirmatory venous test was performed and the newly diagnosed subject was addressed to referral services in order to start treatment. All patients with sexual risk behaviours or with a recent diagnosis of STI were invited to come back after 3-6 months to repeat HIV test.

Participants were asked to fill in an anonymous questionnaire self-administered and semi-structured that and included sociodemographic information, indicators about knowledge of HIV/ STIs, HIV test, condom use, sexual behaviours, perception of risk and stigma, based on the ECDC proposed indicators on behavioural surveillance for migrants and ethnic minorities [11]. The HIV written consent, pre- and post-test counselling questionnaires were translated in 15 different languages.

The pre and post-test counselling involved a staff composed by doctor, nurse, cultural mediator and psychologist, previously trained on HIV/AIDS issues.

Closed questions were dichotomic (true/false) or based on the Likert's scale (from 1 to 5 , established as 1=very low, 2=low, 3 =sufficient, 4=good, 5=excellent).

Migrant status was defined on the basis of citizenship.

Specific pre-test questionnaire items regarding knowledge of HIV/STIS, HIV testing, condom use, perception of risk and stigma were analysed. Distributions of answers were stratified by gender, age group, educational level and immigrant status; Wilcoxon Signed Rank tests and Fisher exact $\chi 2$ tests were used to compare, respectively continuous and categorical data.

\section{Results}

\section{HIV rapid test results}

Figure 1 shows the flow-chart of the study. During the study period, 832 subjects were enrolled. Out of the total sample, $99.2 \%$ 
( $n=825$ ) accepted to undergo the "rapid test". For $68.7 \%$ it was the first test: out of them, $71.4 \%$ were immigrants. 1,000 tests were performed with 1.2 tests/person. Of the 13 HIV positive subjects (prevalence 1.45\%; 95\% Cl 0.75\%-2.53\%), 2 referred to be positive after enrolment and 1 refused the confirmatory venous test. The number of new diagnoses was 10 (incidence $1.22 \%$; $95 \% \mathrm{Cl} 0.58 \%-2.22 \%)$, with one seroconverted. Out of them, 9 were migrants; 5 men who have sex with men (MSM). All of them referred not to have been tested before. Two of them underwent the test for screening and the others performed it because they had an "indicator disease" (such HBV infection, genital wart, tuberculosis). The median CD4 cell count was 347 cell/mmc (range 200-947). Median age was 35.5 (IQR: 27.0-47.0) years, $48.1 \%$ was between 16 and 34 years. $41.7 \%$ of subjects had low educational level.

About $90 \%$ of migrant participants, $28.0 \%$ were from Africa (mainly from Nigeria, Egypt, Senegal and Morocco), $26.0 \%$ from EU countries (Romania and Poland) and 13.6\% from Asia (Afghanistan, Bangladesh). The median length of stay in Italy was 0.7 years (IQR: $0.2-1.2$ ).

HIV-positive individuals were more frequently males and younger than the HIV-negatives. HIV-positivity was particularly elevated among homeless (2.5\%), MSM (18.5\%) and women with female genital cutting (FGC) (25\%) (Data not shown).

\section{Pre-test questionnaire results}

Among the 385 (46.8\%) participants who filled in the pre- questionnaire at baseline, 74 made it after August 2013 and thus the follow-up was not planned. Of the 311 remaining, most of them HIV (81.7\%), only 58 (18.6\%) completed the follow-up.

Table 1 shows the main results of the pre-test questionnaire selected items.

About HIV/AIDS knowledge, $80 \%$ of the total participants knew HIV infection/AIDS: knew about HIV infection (79.7\%) and AIDS (77.9\%), with higher proportions among the higher educated (HIV: $85.7 \%$ vs. $71.4 \%$; AIDS: $83.9 \%$ vs. $69.5 \%$ ) and Italian people (HIV: $93.2 \%$ vs. $76.6 \%$; AIDS: $93.2 \%$ vs. $74.4 \%$ ). Subjects aged $35+$ were more likely to know about AIDS (82.1\% vs. $74.5 \%)$.

Almost all the questions regarding the perception of risk showed worse risk behaviours among migrants. The $76.1 \%$ correctly stated that it is possible to prevent HIV; this proportion significantly increased in Italians and higher educated - Only $22.9 \%$ would use condoms during sexual intercourse (higher for men and younger people). Men also reported higher use of condom during not stable relationships (data not shown).

About HIV test knowledge $75.1 \%$ said that a negative result means "not infected", significantly immigrants $(p=0.041)$ and females $(p=0.005)$. Males $(p=0.055)$ and Italians $(p=0.001)$ know that it could be anonymous and, if negative, could be repeated ( $p=0.02$ and $p=0.001$, respectively).

Specific items were dedicated to the 90 pregnant women. Out of them, 90 pregnant women, $58.8 \%$ reported "missing test " to have "missed the test" due to lack of knowledge (55\%), lack of

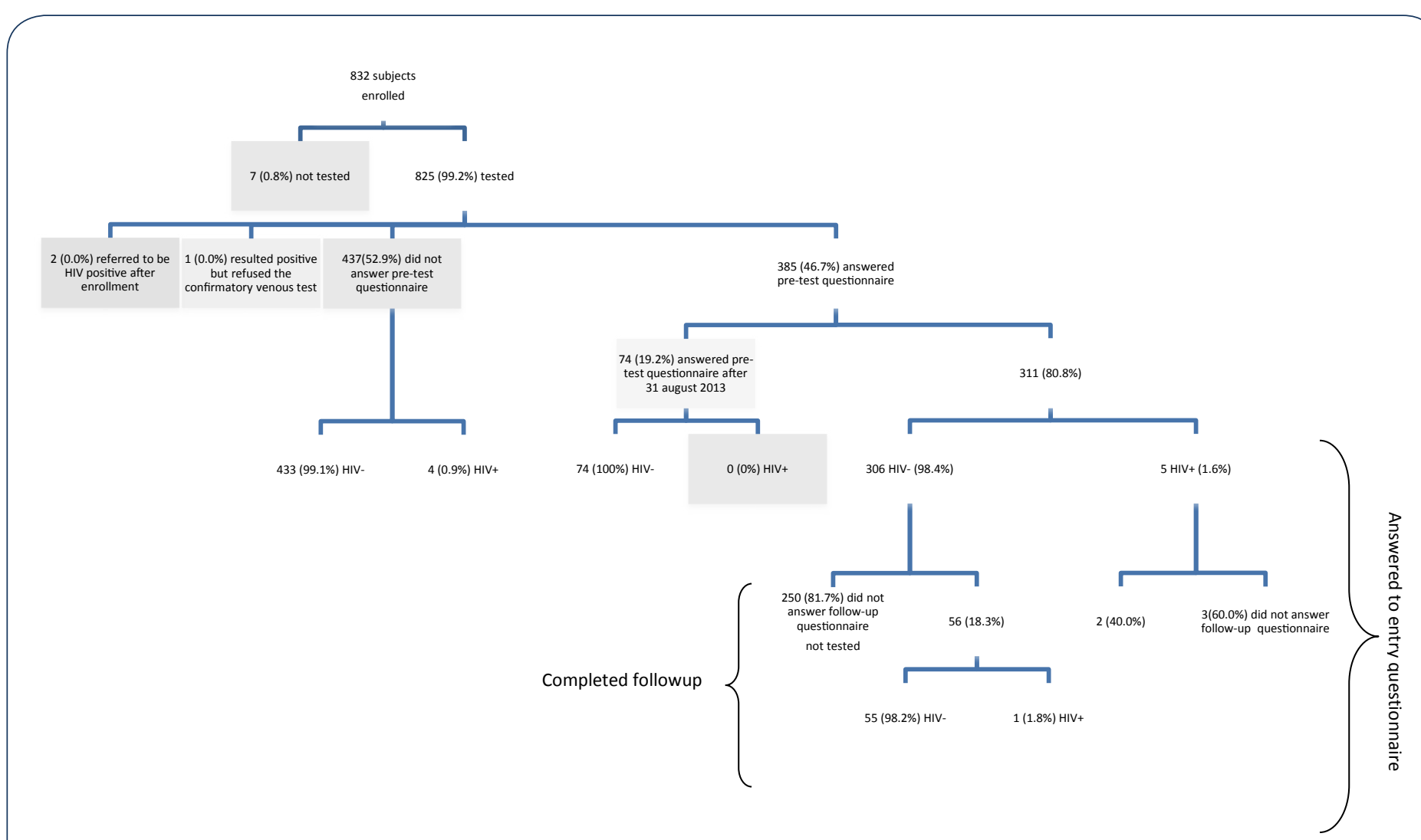

Figure 1 Biochemical markers and tenofovir dosing over time. 


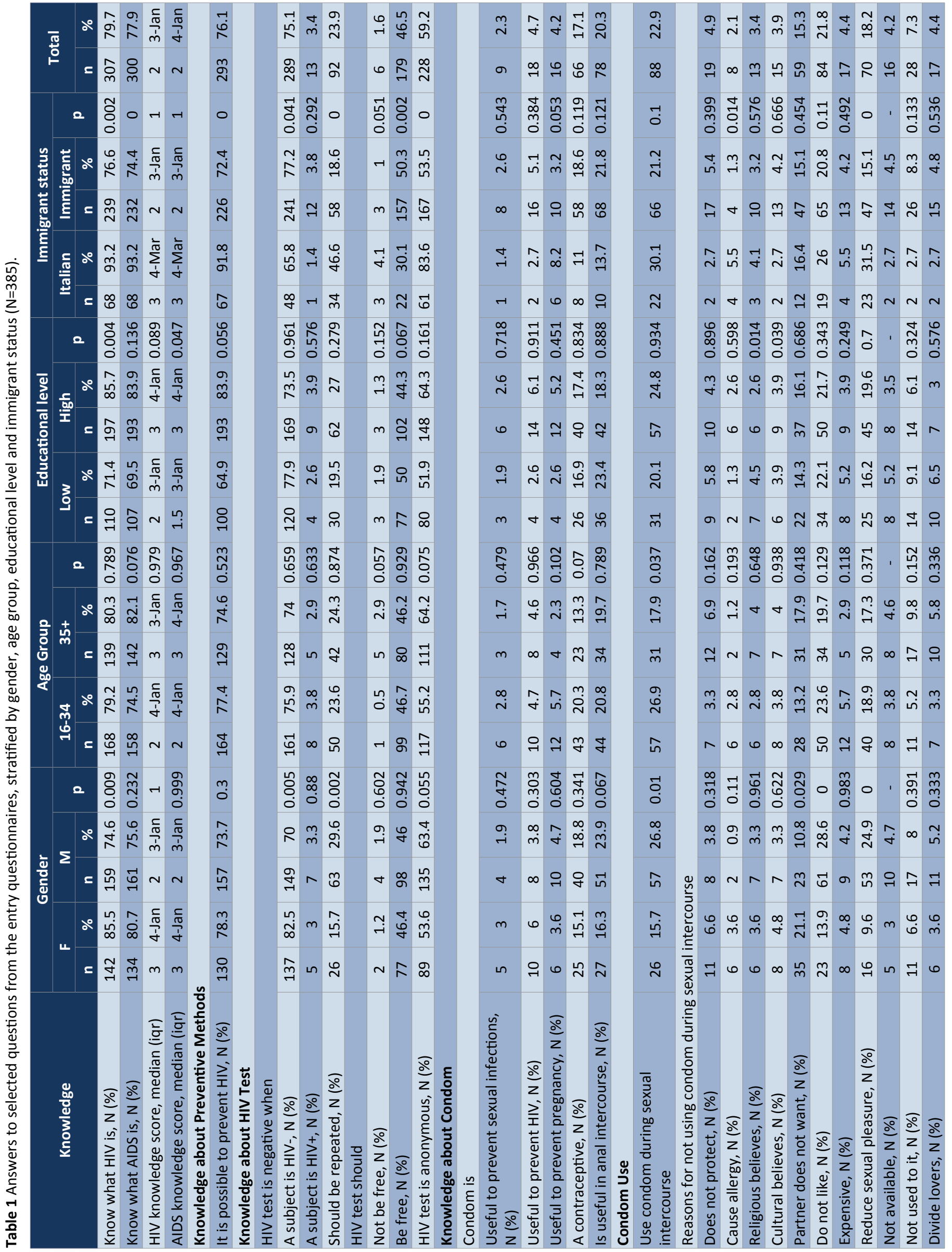




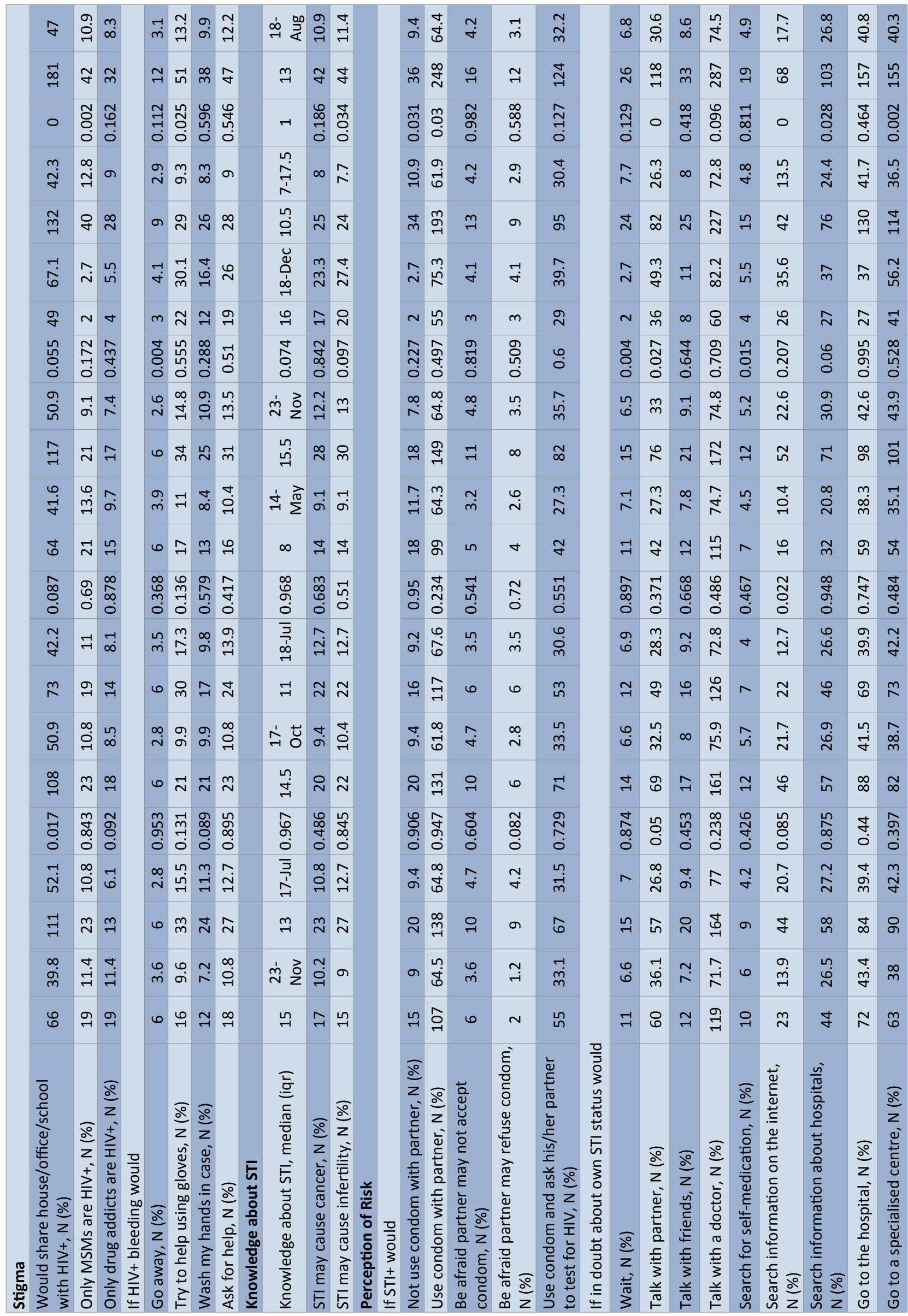


request by the gynaecologists (26.0\%), previous testing (32\%), lack of money to pay the test (13\%) (Data not shown).

About condom knowledge and use, only $22.9 \%$ use it and $4.7 \%$ and $2.3 \%$ affirmed that it can be useful for prevention, respectively against HIV and STIs. They are males $(p=0.010)$ and younger $(p=0.037)$ without difference between migrants and Italians. Among people not using condom, $21.8 \%$ affirmed that they did' not like it, $18.2 \%$ that it reduces sexual pleasure and $15.3 \%$ said it is not accepted by the partner. Inside the females group, almost none know that condom prevent infections and pregnancy.

The proportion of those who answered correctly decreases dramatically investigating STIs knowledge: only $10.9 \%$ affirmed that STIs may cause cancer, and $11.4 \%$ infertility.

When we analysed the perception of risk, only the $32.2 \%$ use condom in presence of an STI and answered that also the partner should be tested. Particularly, females and higher educated talk with partners $(p=0.05$ and $p=0.027$, respectively). However, if in doubt about their STI status, younger search information on internet $(p=0.002)$, the $74.5 \%$ would talk with a doctor, but only $40.8 \%$ would go to the hospital, most of them Italians. As regards stigma, the $10.9 \%$ of respondents affirmed that only MSMs and $8.3 \%$ that only drug addicts can be HIV+, with higher proportions among migrants $(p=0.002)$. Only $47.0 \%$ was willing to share his/ her house/school/office with an HIV+ person, most of them males $(p=0.007)$, Italians $(p=0.001)$ and higher educated $(p=0.005)$.

\section{Discussion}

Despite the decline of the HIV epidemic in Italy, our results confirm the high probability of late presentation among migrants $[18,20]$ never tested before, the largest subgroup inside our vulnerable populations, that still show an high level of acceptability of the rapid test.

Although no cost-effectiveness studies have been performed on the rapid tests, but there have been studies on the evaluation of the effectiveness of the HIV testing as screening [21].

We support the idea that the availability of this kind of testing with high sensibility and specify [22], low costs and high acceptability for specific groups (migrants, homeless, Roma) and at the moment of delivery, should be encouraged at the end to increase access to diagnosis and treatment In fact, all new diagnosed were in-cared.

As in previous studies [23-25], we found poor knowledge about where to get tested for HIV, anonymously and free of charge, and particularly among women. The perception of risk showed some worse behaviour among immigrants.

As reported in a USA study [26], we observed gender differences: in fact males reported condom use more commonly than women during sexual intercourse. The predominant causes of nonuse were attributed to religious believes on lower educated $(p=0.014)$ and cultural believers on higher educated $(p=0.039)$, to pleasure reduction for males $(p=0.001)$ and negotiation for women $(p=0.029)$.
The evaluation of the presence of peer mediators inside the setting was not considered as one of outcomes of the study, because the "health team" consisting of physicians, nurses and specialists is the specific health care approach of the Health, Migration, and Poverty Institute (INMP).

Inside an Italian project conducted on 2010 the care manager figure was added to the others health professionals, serving as a bridge between physicians, specialists, and patients [26]. The central aim of this study was to empower people living with chronic diseases (cardiovascular disease, diabetes, heart failure, and/or at risk of cardiovascular disease) to take a more active role in their health. The "care manager" nurses were trained and they worked directly with individual patients, helping them to make lifestyle changes, monitoring their conditions and providing the necessary information and advice to promote patient empowerment, enhance self-management skills, and achieve better compliance with care recommendations in their self-management skills. The authors reported a positive impact on patient health and self-management and attributed the outcomes to the strong "partnership" between the care manager and the patient and the collaboration between the physician and the care manager. Future studies are needed to be able to assess effectiveness of this method inside the primary health care services for migrant and vulnerable populations.

\section{Limitations of the Study}

The questionnaires data are useful to evaluate the target prevention and treatment service as "data to action", indicated by ECDC. In our study most of participants refused it at the entry of the project and this result could be considered a limitation of the study.

We had similar obstacles in using this instrument as other studies on HIV prevention strategies in the same populations demonstrated to take information about sexual behaviours [27] and to a longitudinal evaluation of counselling effectiveness $[28,29]$.

At the end to understand why half of subjects refused to fill in the questionnaire, we compared both groups by general characteristics. We observed that those who filled in the entry questionnaire were significantly more likely to be Italian, to be younger (median age: 33 vs. 38 years.) and to be highly educated than those who did not fill in the questionnaire. At medical examination those who filled in the entry questionnaire had increased odds of co-infections (OR: 2.31; 95\% Cl: 1.44-3.69) and were more likely to have been exposed to STIs (OR: $1.91 ; 95 \%$ $\mathrm{Cl}$ : 1.14-3.21). Compared to Italians, the odds for immigrants of Northern African origin, Sub-Saharan Africa, Asia, other EU countries and other/not specified origin were respectively $61 \%, 71 \%, 74 \%, 61 \%$ and $76 \%$ lower. Among immigrants, a year increase in the length of stay in Italy was significantly associated with an increase in the OR by $0.9 \%$.

These results strengthen, as already reported, that education level, social inclusion and the state of health at the time of access to the health services, affect the participation of vulnerable populations, including migrants, in public health projects. 
Project timing represented another important study limit, resulting in very low participation to follow-up testing and post-test questionnaire aimed at evaluating changes in sexual behaviours. Low follow-up participation could be related to low perception of risk, maybe associated to the first negative result. Moreover, due to the elusive nature of the target population, some patients moved or forgot the follow up examination despite medical recommendations.

\section{Conclusion}

To obtain targets $90-90-90$, is fundamental to increase the access to health care services and prevention programs for migrants and vulnerable populations, unwires of HIV serostatus. One strategy could be the use of HIV rapid test inside the health care services and prevention programs. In fact we observed a high acceptability of HIV rapid test, as another study described [30]. For this reason, we propose also the availability of rapid HIV testing at the moment of delivery or for example, in Emergency Departments in presence of "indicators diseases.

The approach providing rapid test, that could useful without

\section{References}

1 http://ec.europa.eu/eurostat/statisticsexplained/index.php/ Migration_and_migrant_population_statistics

2 Weine SM, Kashuba AB (2012). Labor migration and HIV risk: A systematic review of the literature. AIDS Behav 16: 1605-1621.

3 Haour-Knipe M (2000). Migration and HIV/AIDS in Europe. In: AIDS Infotheque, pp: 4-14.

4 Rechel B, Mladovsky P, Ingleby D, Mackenbach JP, McKee M (2013) Migration and health in an increasingly diverse Europe. Lancet 381 1235-1245.

5 Prost A, Elford J, Imrie J, Petticrew M, Hart GJ (2008) Social, behavioural, and intervention research among people of sub-Saharan African origin living with HIV in the UK and Europe: Literature review and recommendations for intervention. AIDS Behav 12: 170-194.

6 Vissman AT, Enq E, Aronson RE, Bloom FR, Leichliter JS, et al. (2009). What do men who serve as lay health advisers really do? Immigrant Latino men share their experiences as Navegantes to prevent HIV. AIDS Educ Prev 21: 220-232.

7 7.Del AJ, Bröring G, Hamers FF, Infuso A, Fenton K (2004). Monitoring HIV/AIDS in Europe's migrant communities and ethnic minorities. AIDS 18: 1867-1873.

8 https://ecdc.europa.eu/sites/portal/files/media/en/publications/ Publications/Migrant-health-sexual-transmission.pdf

9 Pharris S, Quinten C, Noori T, Amato-Gauci AJ, van Sighem A, et al. (2015). Estimating HIV incidence and number of undiagnosed individuals living with HIV in the European Union/ European Economic Area, 2015. Euro Surveill, p: 30417.

10 Pharris A, Quinten C, Tavoschi L, Spiteri G, Amato-Gauci AJ, et al. (2015) Trends in HIV surveillance data in the EU/EEA, 2005 to 2014: New HIV diagnoses still increasing in men who have sex with men. Euro Surveill, p: 30071.

11 https://ecdc.europa.eu/sites/portal/files/media/en/publications/ lab and timing barriers, associated to other instruments that reduce the language cultural barriers -such as the presence of trained peer mediators inside the counselling pre and posttest and translation in own languages of written consensus and questionnaires-, could be useful to encourage vulnerable populations to refer to HIV prevention services.

For these reasons, we consider the "rapid test and counselling with a an health team trained "model a new important tool in the framework of the global strategy against AIDS to discover unaware PLHIV, but we agreed with the systematic review of Elwy et al. [31] that compared different interventions for the prevention of HIV/STI: no single intervention could be identified as being more effective than others to change behaviour, increase knowledge or measure an intention to change.

In conclusion, we confirmed that to be migrants and to have low educational level are predictive negative conditions to access to prevention programmes, for which a composite policy can be identified as being more effective to encouraging the marginalised groups to refer to preventions and treatments services.

Publications/0907_TER_Migrant_health_HIV_Access_to_ treatment.pdf

12 Fakoya I, Álvarez-Del Arco D, Monge S, Copas AJ, Gennotte AF, et al. (2016) Advancing migrant access to health services in Europe (AMASE): Protocol for a cross-sectional study. JMIR Res Protoc 5: e74.

13 Burns FM, Johnson AM, Nazroo J, Ainsworth J, Anderson J, et al. (2008) Missed opportunities for earlier HIV diagnosis within primary and secondary healthcare settings in the UK. AIDS 22: 115-22.

14 Evans AR, Parutis V, Hart G, Mercer CH, Gerry C, et al. (2009). The sexual attitudes and lifestyles of London's eastern Europeans (SALLEE Project): Design and methods. BMC Public Health 9: 399.

15 Dray-Spira R, Spire B, Heard I, Lert F, VESPA Study Group (2007). Heterogeneous response to HAART across a diverse population of people living with HIV: Results from the ANRS-EN12-VESPA Study. AIDS 1: S5-12.

16 Fakoya I, Álvarez-Del Arco D, Monge S, Copas AJ, Gennotte AF, et al. (2016) Advancing migrant access to health services in Europe (AMASE): Protocol for a cross-sectional study. JMIR Res Protoc 5: e74.

17 Istituto Superiore Di Sanità (ISS)-Centro Operativo AIDS (CoA) (2016) Aggiornamento delle nuove diagnosi di infezione da HIV e dei casi di AIDS in Italia al 31 Dicembre 2015. Not Ist Super Sanità 29: 3-51.

18 Camoni L., Boros S, Regine V, Santaquilani M, Ferri M, et al. (2015) Aggiornamento delle nuove diagnosi di infezione da HIV e dei casi di AIDS in Italia al 31 dicembre 2014. Notiziario Istituto Superiore di Sanità 28: 3-47.

19 Patel P, Bennett B, Sullivan, T, Parker MM, Heffelfinger JD, et al. (2012) Rapid HIV screening: Missed opportunities for HIV diagnosis and prevention. J Clin Virol 54: 42-47.

20 Carnicer-Pont D, de Olalla PG, Caylă JA, AIDS Working Group (2009) HIV infection late detection in AIDS patients of an European city with increased immigration since mid-1990s. Curr HIV Res 7: 237-243.

21 Long EF, Brandeau ML, Owens DK (2010). The cost-effectiveness and 
population outcomes of expanded HIV screening and antiretroviral treatment in the United States. Ann Intern Med 153: 778-789.

22 https://unitaid.eu/assets/HIV-Rapid-Diagnostic-Tests-for-SelfTesting_Landscape-Report_3rd-edition_July-2017.pdf

23 Mitra D, Jacobsen MJ, O'Connor A, Pottie K, Tugwell P (2006). Assessment of the decision support needs of women from HIV endemic countries regarding voluntary HIV testing in Canada. Patient Educ Couns 63: 292-300.

24 Burns FM, Imrie JY, Nazroo J, Johnson AM, Fenton KA (2007). Why the(y) wait? Key informant understandings of factors contributing to late presentation and poor utilization of HIV health and social care services by African migrants in Britain. AIDS Care 19: 102-108.

25 Lopez-Quintero C, Shtarkshall R, Neumark YD (2005). Barriers to HIV-testing among Hispanics in the United States: Analysis of the National Health Interview Survey, 2000. AIDS Patient Care STDS 19: 672-683.

26 Huang ZJ, Wong FY, De Leon JM, Park RJ (2008). Self-reported HIV testing behaviors among a sample of Southeast Asians in an urban setting in the United States. AIDS Educ Prev 20: 65-77.
27 Ciccone MM, Aquilino A, Cortese F, Scicchitano P, Sassara M, et al. (2010) Feasibility and effectiveness of a disease and care management model in the primary health care system for patients with heart failure and diabetes (Project Leonardo). Vasc Health Risk Manag 6: 297-305.

28 Hoyos J, Fernandez-Balbuena S, de la Fuente L, Sordo L, Ruiz M, et al. (2013). Never tested for HIV in Latin-American migrants and Spaniards: Prevalence and perceived barriers. J Int AIDS Soc 16: 18560.

29 Haour-Knipe M, Fleury F, Dubois-Arber, F (1999). HIV/AIDS prevention for migrants and ethnic minorities: Three phases of evaluation. Soc Sci Med 49: 1357-1372.

30 Loos J, Manirankunda L, Platteau T, Albers L, Fransen K, et al. (2016) Acceptability of a community-based outreach HIV-testing intervention using oral fluid collection devices and web-based HIV test result collection among sub-Saharan African migrants: A mixedmethod study. JMIR Public Health Surveill 2: e33.

31 Elwy R, Hart GJ, Hawkes S, Petticrew M (2002). Effectiveness of interventions to prevent sexually transmitted infections and human immunodeficiency virus in heterosexual men: A systematic review. Arch Intern Med 162: 1818-1830. 